Revista de Matemática: Teoría y Aplicaciones 2002 9(2) : 85-100

CIMPA - UCR - CCSS ISSN: 1409-2433

\title{
APROXIMACIÓN FRACTAL PARA SEMIVARIOGRAMAS FREÁTICOS
}

\author{
J. R. Mercado E. ${ }^{*}$ P. Lázaro Ch. F. Brambila P. ${ }^{* *}$ \\ C. Fuentes R.
}

Received: Jan 2000 / Revised version: Oct 2002

\begin{abstract}
Resumen
Se integra sobre la medida de Hausdorff y se obtiene el exponente Hölder como la codimensión $D_{T}-D$ del fractal, en el espacio Euclidiano en que se encuentra inmerso. Ésto ha resultado de la aplicación de la concepción de integral de Daniell, que posibilita integrar funciones de Lipschitz y de Hölder sobre las medidas de Baire y también, de definir el espacio de fractales con la métrica de Hutchinson.

Se obtiene la potencia para el modelo [potenciado]* de los semivariogramas de procesos estacionarios. Se aplica a los niveles de los mantos freáticos del Valle del Carrizo, Sinaloa, México, y se crean los semivariogramas experimentales y el de ajuste con un modelo potencial, encontrándose que su potencia es $\beta=1,5$. Se obtiene también, que la dimensión fractal de estos mantos es de 2,25.
\end{abstract}

Palabras clave: Fractales, Hölder, codimensión, similaridad, semivariograma, freático.

\begin{abstract}
Hausdorff's measure is integrated upon, and Hölder's exponent is obtained as the codimension $D_{T}-D$ of the fractal in the Euclidian space in which it is immersed. This has resulted from the application of Daniell's integral conception, which makes it possible to integrate Lipschitz's and Hölder's functions into Baire's measures and to define fractal space with Hutchinson's metric.

The power for the potentiated model of the semivariograms of stationary processes is obtained. It is applied to the levels of the phreatic strata of Valle del Carrizo, Sinaloa, Mexico, and the experimental semivariograms, and those of the adjustment with a potential model are created, with the finding that its power is $\beta=1,5$. It is also found that the fractal dimension of these strata is 2,25 .
\end{abstract}

\footnotetext{
* Instituto Mexicano de Tecnología del Agua, (IMTA), Coordinación de Tecnología Hidrológica, Paseo Cuauhnáhuac No. 8532, Progreso, Jiutepec, Morelos, México. Tel/Fax: (52)-(777)-319-35-44, Tel com: 3293600,ext 605 o 532. E-Mail: rmercado@tlaloc.imta.mx

${ }^{* *}$ Facultad de Ciencias, Universidad Nacional Autónoma de México (UNAM), 04510 México, D.F.. tel (5)-6224858. E-Mail: fbp@hp.fciencias.unam.mx
} 
Keywords: fractals, Hölder, codimension, similarity, semivariogram, groundwater.

Mathematics Subject Classification: 28A80; 28A78

\section{Introducción}

Este artículo tiene un doble cometido, por una parte es un reporte de investigación tanto teórica como aplicada, por otra, es docente, porque quiere mostrarle a los estudiantes y estudiosos del tema tanto la totalidad de las estructuras que comprende, como su adaptabilidad a las aplicaciones.

La matemática es la única parte de la ciencia que ha sabido erigirse a través de todos los tiempos, empezando por su etapa empírica, cuando los humanos contaban y observaban los hechos geométricos de su entorno. Siguiendo por la etapa experimental en donde son capaces de realizar mediciones o aforar para construir tablas de datos. Continuando con la etapa analítica en donde majestuosos crearon el álgebra y lograron separarla de la geometría, después construyeron el análisis y las ecuaciones diferenciales, y finalmente, en la última etapa, levantaron el pináculo cuando egregios, fueron capaces de axiomatizar y crear las estructuras de los espacios vectoriales, de los grupos etc.[12], para que Ian Stewart pueda afirmar que la matemática es la ciencia de las estructuras, los patrones y la clasificación.

En este artículo se sigue un camino integral y en sentido inverso al histórico, al partir de la etapa axiomática, seguir con la analítica, luego abordar la experimental de las mediciones y finalmente, rotunda, aplicar a los hechos empíricos de los niveles freáticos.

El tema se ha organizado de la siguiente manera: se retoman los conceptos de las funciones continuas, y los subespacios de las funciones de Lipschitz y las de Hölder, con sus relaciones de embebimientos compactos. Se repasan los conceptos de conjuntos y medidas de Baire, como concepción débil de los de Borel. Se estudia la integral de Daniell aplicada a las funciones de Lipschitz y Hölder sobre las medidas de Baire y las de Hausdorff.

Se aplica a la aproximación de un grafo fractal por un fractal autosimilar y se obtiene el exponente Hölder como la codimensión del fractal en el espacio Euclidiano en el que se encuentra inmerso.

Se repasan los conceptos de procesos estacionarios y sus semivariogramas. Se aplican a los niveles de los mantos freáticos y se obtienen, por mínimos cuadrados, los parámetros de los estimados. Se representan gráficamente los semivariogramas y los niveles de los mantos freáticos.

Por último parece pertinente recordar que la importancia de estudiar las funciones continuas no diferenciables, surge desde distintos campos de las aplicaciones, desde la mecánica cuántica, el movimiento Browniano, las curvas de evolución económica, los fenómenos hidrológicos y geológicos, los procesos en los medios porosos, etc.

\section{Funciones continuas}

Una función define una correspondencia entre los elementos de dos espacios, de tal manera que se establece una relación entre el argumento en el primer espacio y su imagen única en el segundo $f: \Omega \longrightarrow X$. En general los dos espacios tienen estructura de espacios topológicos; en donde se forma una pareja $\left(\Omega, \mathcal{T}_{\Omega}\right)$, compuesta por un conjunto $\Omega$, y una 
colección de sus subconjuntos $\mathcal{T}_{\Omega}$. A estos se les llama abiertos, y satisfacen las condiciones: 1.) El conjunto total $\Omega$ y el conjunto vacío $\emptyset$, están en la colección, 2.) La colección es cerrada bajo intersecciones finitas, y 3.) La colección es cerrada bajo uniones arbitrarias.

Es importante el caso cuando $\Omega$ es un espacio métrico, en donde la topología se determina a partir de las bolas abiertas, y además es completo, cuando las sucesiones fundamentales de Bolzano o de Cauchy son convergentes.

Si $X$ es además un espacio lineal, las funciones conforman también un espacio lineal, al definir la suma de funciones y la multiplicación por escalar en forma puntual. Las funciones frecuentemente estan definidas sólo en un subconjunto del espacio $\Omega$, llamado dominio, y es relievante el caso cuando este subconjunto es un compacto del espacio $\Omega$. Una situación que se encuentra con bastante amplitud es la de las funciones continuas. Una de las ventajas de la continuidad radica en que las funciones continuas alcanzan el supremo en un dominio compacto.

Ubicados en el espacio de funciones continuas con dominios en un compacto y con imagenes reales, la distancia se define por la norma del supremo $\|f\|=\sup _{t \in \Omega}\|f(t)\|$. Como en el caso de las funciones de variación acotada, se realiza una partición del dominio por subintervalos compactos y se evalúa la variación en cada uno de los subintervalos $V_{t_{1}}^{t_{2}}(f)=\sup _{t, s \in\left[t_{1}, t_{2}\right]}\|f(t)-f(s)\|$.

Sin embargo, conviene también abordar la función desde el punto de vista de su gráfica o grafo $\Gamma_{f}=\left\{(t, f(t)) \in \mathbb{R}^{D_{T}+1}: t \in \Omega\right\}$. Dentro de los subespacios de las funciones continuas, uno de los que pueden destacarse es el subespacio de las funciones de Lipschitz, en donde las secantes entre dos puntos del grafo tienen, localmente, pendientes acotadas,

$$
V_{t_{1}}^{t_{2}}(f)=\sup _{t, s \in\left[t_{1}, t_{2}\right]}|| f(t)-f(s)|| \leq \sup _{t, s \in\left[t_{1}, t_{2}\right]} c|t-s| \leq c\left|t_{2}-t_{1}\right|
$$

y en particular, las funciones continuas de Hölder

$$
V_{t_{1}}^{t_{2}}(f)=\sup _{t, s \in\left[t_{1}, t_{2}\right]}\|f(t)-f(s)\| \leq \sup _{t, s \in\left[t_{1}, t_{2}\right]} c|t-s|^{\alpha} \leq c\left|t_{2}-t_{1}\right|^{\alpha} .
$$

\subsection{Funciones de Hölder}

Cuando $\Omega$ es un compacto en $\mathbb{R}$ y $\alpha$ un parámetro real en el intervalo $(0,1]$, las funciones definidas con dominio en $\Omega$ y a valor $\mathbb{R}^{D_{T}}$ que satisfacen la desigualdad

$$
\|f(t)-f(s)\| \leq c|s-t|^{\alpha},
$$

con $c$ una constante positiva, que depende de $f$ pero no de $t$ ni de $s$, conforman un subespacio lineal del espacio de funciones continuas $C^{0}(\Omega)$ llamado espacio de Hölder y se denota por $C^{0, \alpha}(\Omega)$. Estas funciones en $C^{0, \alpha}(\Omega)$ son por supuesto, uniformemente continuas en $\Omega$, [2].

Este subespacio admite un embebimiento compacto en el espacio de las funciones continuas, en el sentido de que la aplicación que define el embebimiento transforma conjuntos acotados en conjuntos cuya clausura es compacta entre los espacios de Banach, en tanto que en los espacios de Hilbert transforma sucesiones (redes) débilmente convergentes en fuertemente convergentes. Este embebimiento expresa el teorema de Arzelá-Ascoli:

$$
C^{0, \alpha}(\Omega) \hookrightarrow C^{0}(\Omega)
$$


El conjunto de estas funciones, resulta ser un espacio de Banach, con la norma definida por

$$
\|f\|_{\alpha}=\sup _{t \in \Omega}\|f(t)\|+\sup _{t, s \in \Omega, t \neq s} \frac{\|f(s)-f(t)\|}{|s-t|^{\alpha}}
$$

y en general, cuando el exponente $\alpha$ se incrementa se reduce el espacio, pero puede definirse un embebimiento compacto.

\section{Conjuntos de Baire}

Se conforma una nueva pareja con el mismo espacio total $\Omega$, y una nueva colección de subconjuntos $\mathcal{A}$, que satisfacen: 1.) El conjunto total $\Omega$ y el conjunto vacío $\emptyset$, están en la colección, 2.) Si un conjunto está, también está su complemento, 3.) Si se tiene una cantidad numerable de conjuntos en la colección, la unión de ellos, también permanece en la colección. A la colección $\mathcal{A}$ se le llama una $\sigma$-álgebra.

En el espacio métrico completo $\Omega$, puede estructurarse un nuevo sistema de subconjuntos que amplia los de la topología, éste se conforma como la más pequeña $\sigma$-álgebra que contiene a los abiertos y por tanto también a los cerrados. Se tiene así la $\sigma$-álgebra de Borel. Los conjuntos compactos son los cerrados totalmente acotados y por tanto forman parte de esta nueva estructura de los subconjuntos del espacio $\Omega$.

Dentro de la $\sigma$-álgebra de los borelianos, existe otra $\sigma$-álgebra de los conjuntos de Baire, ellos son conjuntos $G_{\delta}$-compactos, es decir una colección numerable de abiertos cuya intersección es compacta, [10],

$$
B=\bigcap_{n=1}^{\infty} B_{n} .
$$

Una $\sigma$-álgebra es el dominio de definición para una medida como una función que satisface: 1.) la medida del vacío es el cero: $\mu(\emptyset)=0,2$.) Los conjuntos de la colección tiene medida positiva o a lo menos medida cero, $\mu(A) \geq 0, \forall A \in \mathcal{A}$, 3.) la medida es contablemente aditiva, en el sentido de que si $\left(A_{n}\right)_{n}$ es una colección numerable y disyunta de subconjuntos en la $\sigma$-álgebra $\mathcal{A}$, la imagen de la unión es la serie de las imagenes: $\mu\left(\bigcup^{*} A_{n}\right)=\sum_{n} \mu\left(A_{n}\right)$.

Se considera ahora el caso más específico de un espacio métrico compacto. Sobre la $\sigma$-álgebra de Baire se definen las medidas de Baire normalizadas. Luego puede pensarse en la integración sobre las más pequeñas de las de Hölder que son las de Lipschitz, luego sobre las funciones de Hölder, posteriormente también sobre las continuas, y finalmente sobre las funciones medibles.

Por el teorema de Caratheodory, las medidas de Baire tienen una única extensión a una medida de Borel regular. Así que las medidas de Baire, constituyen un concepto débil muy útil para las medidas de Borel.

\section{Integral de Daniell}

Dentro de la concepción de integral de Daniell se considera un retículo vectorial $\mathcal{R}$, el cual es una clase de funciones acotadas a valor real, cerrado bajo las operaciones de suma, 
multiplicación por escalar, el máximo de dos funciones o suma lógica y el mínimo de dos funciones o producto lógico. [3], [14].

La clase de funciones de Hölder, como subespacio satisfacen las dos condiciones de ser cerradas bajo la suma y multiplicación por escalar y son acotadas en tanto que funciones continuas definidas en un compacto.

Se demuestra que satisface también la condición de ser cerradas bajo las sumas lógicas; y como la suma de la suma lógica con el producto lógico es la suma usual de funciones, se deduce que satisfacen también la propiedad de ser cerradas bajo el producto lógico. Estos resultados simbólicamente se resumen en el siguiente cuadro:

$$
f, g \in C^{0, \alpha}(\Omega), a \in \mathbb{R} \Rightarrow\left\{\begin{array}{l}
f+g \in C^{0, \alpha}(\Omega) \\
a f \in C^{0, \alpha}(\Omega) \\
(f \vee g)(t)=\operatorname{máx}\{f(t), g(t)\} \in C^{0, \alpha}(\Omega) \\
(f \wedge g)(t)=\operatorname{mín}\{f(t), g(t)\} \in C^{0, \alpha}(\Omega)
\end{array}\right.
$$

En particular, también el valor absoluto de una función esta en la clase, ya que es la suma lógica de la función y su opuesta $|f|=f \vee(-f)$, la cual puede servir también de punto de partida para definir las dos operaciones lógicas del retículo vectorial.

En consecuencia, las funciones en $C^{0, \alpha}(\Omega)$, constituyen un retículo vectorial en donde puede definirse la integral de Daniell, la cual es una funcional definida en el retículo vectorial con las propiedades:

1. Es lineal: para cualquier par de escalares $a$ y $b$, y cualquier par de funciones en el retículo $f$ y $g$, se tiene la linealidad usual de la integral: $\varphi(a f+b g)=a \varphi(f)+b \varphi(g)$.

2. Es monótona: Las funciones positivas tiene integral positiva, en el sentido de que si $f$ es una función en el retículo vectorial y es $f \geq 0$, entonces la integal también preserva la desigualdad $\varphi(f) \geq 0$ o bien: $f \geq 0 \Rightarrow \varphi(f) \geq 0$.

3. Es continua en cero: Si $\left(f_{i}\right)_{i}$ es una sucesión monodecreciente de funciones en el retículo vectorial que convergen puntualmente a cero, entonces la integral también preserva la convergencia a cero de la sucesión de integrales: $f_{i} \geq f_{i+1},\left(f_{i}\right)_{i} \stackrel{i}{\rightarrow} 0 \Rightarrow$ $\left(\varphi\left(f_{i}\right)\right)_{i} \stackrel{i}{\rightarrow} 0$.

Los límites de las sucesiones de funciones monócrecientes del retículo vectorial, permiten extender la integral de Daniell, a la clausura del retículo. Por el embebimiento compacto, la integral considerada se extiende de las funciones de Lipschitz a las Hölder, y de éstas a las funciones continuas.

En el espacio de medidas se define la distancia de Hutchinson

$$
d_{H}(\mu, \nu)=\sup \left\{\int_{\Omega} f d \mu-\int_{\Omega} f d \nu: f \in \operatorname{Lip}\right\},
$$

con ésta métrica el espacio de las medidas definidas sobre los borelianos del espacio métrico compacto, resulta ser también un espacio métrico completo y se le conoce como el espacio de los fractales. 
Por un sistema de funciones iteradas se entiende una colección finita de contracciones $\left(w_{i}\right)_{i \in\{1, N\}}$ cada una con su factor de contracción $\left(\frac{1}{q_{i}}\right)_{i}$ definidas sobre el espacio métrico $\Omega$ y un sistema de probabilidades $\left(p_{i}\right)_{i}$ :

$$
\left\{\Omega ;\left(w_{i}, \frac{1}{q_{i}}, p_{i}\right)_{i \in\{1, N\}}\right\}
$$

en donde las resoluciones se representan por $q_{i}$ y el número de rasgos por $N$. Sobre el espacio de fractales se define el operador de Márkov, asociado a un sistema de funciones iteradas, [1], [7], como la medida:

$$
M(\nu)=\sum_{i=1}^{N} p_{i} \nu \circ w_{i}^{-1}
$$

este operador resulta ser una contracción con factor de contracción $s=\bigvee_{i=1}^{N} \frac{1}{q_{i}}$ y por el teorema del punto fijo de Banach tiene como atractor un único punto fijo conocido como la medida invariante del operador de Márkov: $M(\mu)=\mu$. En $\mathcal{H}(\Omega)$, el espacio de los soportes de estas medidas invariantes, se considera la aplicación definida por

$$
W: \mathcal{H}(\Omega) \rightarrow \mathcal{H}(\Omega), W(F)=\bigcup_{i=0}^{N} w_{i}(F), \forall F \in \mathcal{H}(\Omega),
$$

resulta ser una aplicación contraída con el mismo factor de contracción $s$, y a su único punto fijo $A \in \mathcal{H}(\Omega)$, se le llama el atractor del sistema de funciones iteradas, y es tal que

$$
A=W(A)=\bigcup_{i=0}^{N} w_{i}(A), \text { y } A=\lim _{n \rightarrow \infty} W^{\circ n}(F), \forall F \in \mathcal{H}(\Omega),
$$

en tanto que se conoce como operador de Barnsley, a la aplicación [7],

$$
\lim _{n \rightarrow \infty} W^{\circ n}(F): \mathcal{H}(\Omega) \rightarrow \mathcal{H}(\Omega)
$$

\subsection{Integral de Daniell y Medida de Hausdorff}

La medida de Hausdorff se define en la $\sigma$-álgebra de los conjuntos de Baire, y se extiende a los conjuntos de Borel. Al tener la integral como funcional lineal, que sin pérdida de generalidad puede considerarse positiva, existe la $\sigma$-álgebra en $\Omega$ de los conjuntos de Baire, y también existe una única medida de Baire positiva, definida en esa $\sigma$-álgebra, que permite representar la funcional como

$$
\varphi(f)=\int_{\Omega} f d \mu
$$

y por el teorema de Caratheodory, esta medida se extiende de manera única a una medida de Borel regular, definida en todos los borelianos de $\Omega$. Esta última medida es, salvo posiblemente una constante, la medida de Lebesgue de $\mathbb{R}^{D_{T}}$ y en tanto que dimensión entera es, salvo una constante multiplicativa, la medida de Hausdorff, por tanto renormalizando la funcional $\varphi$ de la integral de Daniell, puede suponerse que $\mu$ es la medida de Hausdorff, $[13]$. 


\section{Aproximación fractal}

Se considera el grafo de alguna función obtenida experimentalmente

$$
\Gamma_{f}=\left\{(t, f(t)) \in \mathbb{R}^{D_{T}+1}: t \in \Omega\right\},
$$

se define una malla de cubos elementales que depende de la resolución y del paso en el proceso de fractalización.

Dado un número real $q>1$ y un entero positivo $n \geq 1$, la resolución se define por $\left(\frac{1}{q}\right)^{n}$. Se consideran los cubos dependientes de esta resolución. Sea la malla $M_{q, n}$ de los puntos de $\mathbb{R}^{D_{T}}$ cuyas coordenadas son múltiplos enteros de la resolución $\left(\frac{1}{q}\right)^{n}$ y la colección $\left(C_{q, n}(a)\right)_{n}$ de todos los cubos con vértices en la malla $M_{q, n}$ con $a \in M_{q, n}$, estos cubos pueden describirse como

$$
C_{q, n}(a)=\left\{\begin{array}{c}
x \in \mathbb{R}^{D_{T}}: a^{i}=k\left(\frac{1}{q}\right)^{n} \leq x^{i} \leq(k+1)\left(\frac{1}{q}\right)^{n}, \\
\exists k \in \aleph, 1 \leq i \leq D_{T}+1
\end{array}\right\}
$$

y aquellos cubos que intersectan el grafo $\Gamma_{f}$ constituyen la subcolección, [4],

$$
\left(C_{q, n}^{\prime}(a)\right)_{n}=\left(C_{q, n}(a) \cap \Gamma_{f}\right)_{n}
$$

Con el objeto de estimar la integral, se considera el punto $a$ en la malla $M_{q, n}$ y el intervalo $\left[a^{i}, a^{i}+\left(\frac{1}{q}\right)^{n}\right]$. El rango de $f$ se estima por el número de cubos que intersectan el grafo multiplicado por el alto de los cubos $\left(\frac{1}{q}\right)^{n}$. Cuando $n$ es un número suficientemente grande, la resolución se hace pequeña y $q^{n}$ por el contrario, llega a ser suficientemente grande. Sea $m$ el número de cubos en la dirección horizontal, éste puede representarse por el entero más pequeño que supera el valor $q^{n}$. La integral se estima por

$$
\begin{gathered}
\int_{C_{q, n}^{\prime}(a)} f(t) d \mu(t) \leq\left.\sum_{k=0}^{m-1} c|t-s|^{\alpha}\right|_{\left[a^{i}, a^{i}+\left(\frac{1}{q}\right)^{n}\right]} \int_{\left[a^{i}, a^{i}+\left(\frac{1}{q}\right)^{n}\right]} d \mu(t) \\
\leq m c\left(\frac{1}{q}\right)^{n \alpha}\left(\frac{1}{q}\right)^{n} \leq c\left(\frac{1}{q}\right)^{-n}\left(\frac{1}{q}\right)^{n \alpha}\left(\frac{1}{q}\right)^{n}=c\left(\frac{1}{q}\right)^{n \alpha} .
\end{gathered}
$$

De otra parte, con $N_{q, n}^{\prime}$ representando el número de cubos que intesectan el grafo de la función, la integral puede evaluarse como

$$
\int f(t) d \mu(t)=\left(\frac{1}{q}\right)^{n\left(D_{T}-1\right)} N_{q, n}^{\prime}\left(\frac{1}{q}\right)^{n}=N_{q, n}^{\prime}\left(\frac{1}{q}\right)^{n D_{T}} .
$$

Combinando los dos estimados, se obtiene entonces que

$$
N_{q, n}^{\prime}\left(\frac{1}{q}\right)^{n D_{T}} \leq c\left(\frac{1}{q}\right)^{n \alpha}, \mathrm{o}, N_{q, n}^{\prime} \leq c\left(\frac{1}{q}\right)^{n\left(\alpha-D_{T}\right)}
$$

por tanto

$$
-r=\alpha-D_{T}, \mathrm{o}, \alpha=D_{T}-r
$$


Debido a la monotonía de la dimensión de Hausdoff, expresada por la correlación

$$
F \subseteq F^{\prime} \Rightarrow \operatorname{dim}_{H} F \leq \operatorname{dim}_{H} F^{\prime}
$$

se concluye que el fractal del grafo de la función es aproximado superiormente por un fractal autosimilar de dimensión de Hausdorff $r=D_{T}-\alpha$; o bien, puede decirse que el exponente de Hölder es la codimensión $D_{T}-r$.

\section{Procesos estocásticos}

A la pareja de un espacio topológico y una $\sigma$-álgebra de sus subconjuntos, $(\Omega, \mathcal{A})$, se le llama espacio muestral, y en un contexto probabilístico, se le conoce como espacio de eventos elementales. En este espacio se define una medida de probabilidad la cual es una función que toma sus argumentos en la $\sigma$-álgebra y sus valores o imagenes en los reales y satisfacen además de las condiciones de una medida, la condición de normalización: la medida del espacio es finita y se normaliza a la unidad $\mu(\Omega)=1$; se tiene así la tripla $(\Omega, \mathcal{A}, \mu)$ llamada espacio de probabilidad.

Sea ahora otro espacio de topológico $\left(X, \mathcal{T}_{X}\right)$ y el espacio muestral correspondiente, constituido por el conjunto $X$ y una $\sigma$-álgebra $\mathcal{B}$; a la pareja $(X, \mathcal{B})$ se le conoce como espacio fásico.

Se define una variable aleatoria $\xi$ como una función entre estos espacios muestrales

$$
(\Omega, \mathcal{A}) \longrightarrow(X, \mathcal{B}), \omega \longmapsto \xi(\omega)
$$

que debe ser medible en el sentido de que la imagen inversa aplica los conjuntos medibles de $X$ en los conjuntos medibles de $\Omega$,

$$
\xi^{-1}(\mathcal{B}) \subseteq \mathcal{A}
$$

A esta variable aleatoria se le asocia una distribución de (probabilidad), definida a través de la medida de probabilidad como

$$
F_{\xi}(B)=\mu[\omega: \xi(\omega) \in B], o, F_{\xi}=\mu \circ \xi^{-1},
$$

la cual también es una medida de probabilidad en el espacio muestral $(X, \mathcal{B})$, y lo hace un espacio de probabilidad coordinado con el primero. Las variables aleatorias y sus distribuciones asociadas pueden indexarse, considerando una colección de variables aleatorias $\left(\xi_{t}\right)_{t \in T}$, y conformando de esta manera, lo que se conoce como un proceso estocástico

$$
T \times \Omega \longrightarrow X,(t, \omega) \longmapsto \xi(t, \omega)
$$

para valores del parámetro $t$ en determinado intervalo $T$, e imagenes en el espacio muestral fásico $(X, \mathcal{B})$.

Con $k=1,2 \ldots$, se definen para la variable aleatoria $\xi$ los momentos y los momentos centrales por

$$
E\left(\xi^{k}\right)=\int x^{k} d F_{\xi}(x), E(\xi-E(\xi))^{k}=\int(x-E(\xi))^{k} d F_{\xi}(x),
$$


la función de correlación (o autocovarianza) para el proceso estocástico $(\xi(t))_{t \in T}$ por

$$
B(t, s)=E((\xi(t)-E \xi(t)) \overline{(\xi(s)-E \xi(s))}),
$$

cuando existe $E|\xi(t)|^{2}$, para $t \in T$ y la función de covariación por

$$
C(t, s)=E \xi(t) \overline{\xi(s)}
$$

\subsection{Procesos Estacionarios}

Se conocen como procesos estacionarios en amplio sentido, aquellos procesos estocásticos en que la media o esperanza matemática es una constante independiente del parámetro $t$ y la función de correlación sólo depende de la diferencia de los parámetros, [6],

$$
E \xi(t)=m=a=c t e, B(t, s)=B(t-s), B(t)=B(t, 0) .
$$

El semivariograma se define como la mitad de la varianza

$$
\gamma(s, t)=\frac{1}{2} E(\xi(s)-\xi(t)) \overline{(\xi(s)-\xi(t))}
$$

y sí se considera la variable aleatoria relativa

$$
\tilde{\xi}_{t, h}=\xi(s)-\xi(t)=\xi(t+h)-\xi(t),
$$

el semivariograma es la mitad de la varianza de la variable aleatoria relativa y resulta ser función del incremento $h$ :

$$
\gamma(t+h, t)=\frac{1}{2} E(\tilde{\xi}(t ; h)) \overline{(\tilde{\xi}(t ; h))}
$$

Como se usa generalmente en trabajos de campo, el parámetro no se representa por la variable $t$ sino por las posiciones en donde se realiza el muestreo, de tal manera que las distintas posiciones de medición sirven de parámetro para la variable aleatoria en observación, conviene ergo establecer la correspondencia

$$
t \longmapsto x_{t}=x_{i}, i=1,2, \ldots, t+h \longmapsto x_{t+h}=x_{i}+h .
$$

El semivariograma expresa matemáticamente la manera en que la variancia de la variable aleatoria relativa cambia con la separación de los puntos, [11].

\subsubsection{Semivariogramas}

El semivariograma experimental puede calcularse con el estimador clásico siguiente:

$$
\hat{\gamma}(h)=\frac{1}{2 N(h)} \sum_{i=1}^{N(h)}\left[\xi\left(x_{i}+h\right)-\xi\left(x_{i}\right)\right]^{2},
$$

donde $N(h)$ es el número de pares de puntos separados por una distancia $h$. 
El semivariograma experimental generalmente es representado por un modelo teórico para facilitar el cálculo de la matriz de covariancia, [5]. De entre estos modelos se distinguen: el esférico, el exponencial, el gaussiano y el potencial. Para escalar los semivariogramas de los mantos freáticos se utiliza un modelo que satisfaga:

$$
\gamma(r h)=r^{\beta} \gamma(h)
$$

donde $r$ es el factor de escala y $\beta$ una potencia positiva. La función que satisface esta propiedad, con $c>0, \mathrm{y} \beta>0$, es una función potencial del tipo:

$$
\gamma(h)=c h^{\beta},
$$

Cuando la variable aleatoria depende de una sola variable independiente $(x, \xi) \in \mathbb{R}$, la relación entre $\beta$ y la dimensión fractal $D$ es: $\beta=4-2 D$, [4, Falconer]. De otra parte, la generalización de ese resultado, cuando la variable aleatoria depende de $(n-1)$-variables independientes $\left(x_{1}, x_{2}, \ldots, x_{n-1}, \xi\right) \in \mathbb{R}^{n}$, con $n$ la dimensión topológica o de Euclides; como se ha demostrado en el presente trabajo (17), es:

$$
\beta=2 \alpha=2\left(D_{T}-D\right)=2(n-D),
$$

luego, con $D_{T}=n=3$, puesto que $(x, y, h) \in \mathbb{R}^{3}$, la dimensión fractal de los niveles de los mantos freáticos en un mes determinado es

$$
D=D_{T}-\frac{1}{2} \beta=3-\frac{1}{2} \beta .
$$

Los parámetros c y $\beta$ de la ecuación (29) pueden obtenerse con el método de mínimos cuadrados.

\section{Aproximación por mínimos cuadrados}

Este método, creado por Legendre, suscitó algunos malentendidos y conatos de enfrentamiento con Gauss por su autoría. Del triángulo rectángulo, estudiado por los antiguas culturas de Mesopotamia, China, la India y Grecia, emergió el concepto de ortogonalidad que evolucionó hasta surgir, egregía, la estructura de espacios de Hilbert, creada por Schmidt, en cuyo contexto se explica de manera muy clara, muchos procesos de aproximación como el que nos ocupa.

De una colección de $m$ datos se forma un $m$-vector columna. Una $m \times n$-matriz con $n$ columnas linealmente independientes, representa la transformación lineal de los parámetros a los datos. Con los vectores columnas de la matriz se conforma un subespacio lineal cerrado de dimensión $n$. Se proyecta el vector de datos sobre este subespacio, y resulta que de acuerdo con el teorema de la proyección de los espacios de Hilbert, esta proyección determina el punto de mejor aproximación entre los datos y los parámetros buscados. Sí $A$ es la matriz, con columnas $A_{j}, x$ los parámetros, $\hat{x}$ el parámetro que resulta de la proyección de los datos $y$. El vector $y-\hat{x}$ es ortogonal a todos los vectores columnas de $A, A_{j}$; y el parámetro proyección $\hat{x}$ se expresa como combinación lineal de los vectores columnas de $A$, que generan al subespacio cerrado $M$, [8],

$$
\begin{aligned}
\left\langle y-\hat{x}, A_{i}\right\rangle & =0 \\
\hat{x} & =\hat{x}^{j} A_{j}
\end{aligned}
$$


de este par de criterios resultan las ecuaciones llamadas normales

$$
A^{T} A \hat{x}=A^{T} y
$$

luego la aproximación es

$$
\hat{x}=\left(A^{T} A\right)^{-1} A^{T} y
$$

la cual minimiza la distancia de los datos al parámetro proyección

$$
\min _{x \in M}\|y-A x\|=\|y-\hat{x}\|
$$

entonces para el caso presente, con los elementos de la matriz simétrica dados por los semivariogramas de las parejas de puntos y la condición de insesgabilidad, y los datos dados por los semivariogramas experimentales, se busca el parámetro proyección minimizador de la suma de los cuadrados del error:

$$
\operatorname{SCE}(\mathbf{c} ; \beta)=\sum_{j=1}^{M} \sum_{i=1}^{N}\left[\gamma\left(h_{i . j}, c_{j}, \beta_{j}\right)-\hat{\gamma}_{i, j}\right]^{2},
$$

donde $i=1, \ldots, N, N$ es el número de puntos del semivariograma, $j=1, \ldots, M, M$ es el número de meses; $\mathbf{c}=\left(c_{1}, \ldots, c_{M}\right)$, y $\beta=\left(\beta_{1}, \ldots, \beta_{M}\right)$. El número de incógnitas es $2 M$.

La estimación de los $M+1$ parámetros $\left(c_{1}, c_{2}, \ldots, c_{M}, \beta\right)$, se simplifica considerablemente con la minimización de la diferencia cuadrática de los logaritmos de los semivariogramas, es decir con la minimización de:

$$
S C E(\mathbf{c} ; \beta)=\sum_{j=1}^{M} \sum_{i=1}^{N}\left[\ln \gamma\left(h_{i . j}, c_{j}, \beta_{j}\right)-\ln \hat{\gamma}_{i, j}\right]^{2} .
$$

La introducción de la ecuación (29) en la ecuación (36), y la aplicación del método de mínimos cuadrados conduce a las siguientes expresiones, donde $x=\ln h, \mathrm{y}, y=\ln \hat{\gamma}$ :

$$
\begin{gathered}
\beta=\frac{\sum_{j=1}^{M} \sum_{i=1}^{N} x_{i, j} y_{i, j}-\left[\sum_{j=1}^{M}\left(\frac{1}{N} \sum_{i=1}^{N} x_{i, j} \sum_{i=1}^{N} y_{i, j}\right)\right]}{\sum_{j=1}^{M} \sum_{i=1}^{N} x_{i, j}^{2}-\sum_{j=1}^{M}\left[\frac{1}{N}\left(\sum_{i=1}^{N} x_{i, j}\right)^{2}\right]}, \mathrm{y} \\
\ln \left(c_{j}\right)=\frac{1}{N}\left[\sum_{i=1}^{N}\left(y_{i, j}-\beta x_{i, j}\right)\right] .
\end{gathered}
$$

\section{Observaciones}

Las observaciones se realizaron en el Distrito de Riego 076, Valle del Carrizo, Sinaloa, el cual tiene una superficie de 43,259 ha, se ubica en la parte Norte del Estado de Sinaloa y abarca los municipios de Ahome y El Fuerte. Geográficamente se ubica entre las coordenadas $26^{\circ} 05$ y $26^{\circ} 22$ de latitud norte y $108^{\circ} 53$ y $109^{\circ} 00$ de longitud oeste. Limita al 
norte con el Estado de Sonora, al este y sur con la Sierra Madre Occidental y al oeste con el Golfo de California.

La red de pozos de observación freatimétrica tiene un total de 167 pozos con una profundidad de $2.0 \mathrm{~m}$ y se distribuyen en el área según la figura (1), la densidad aproximada es de un pozo por 260 ha. La toma de lecturas de la profundidad del nivel freático se realiza a intervalos de un mes utilizando el método de la manguera. El registro histórico de las observaciones de la profundidad del nivel freático disponible en el distrito es de cinco años (1992-1996). Las observaciones de estos niveles son útiles para realizar un análisis con fines de caracterización o modelación del sistema.

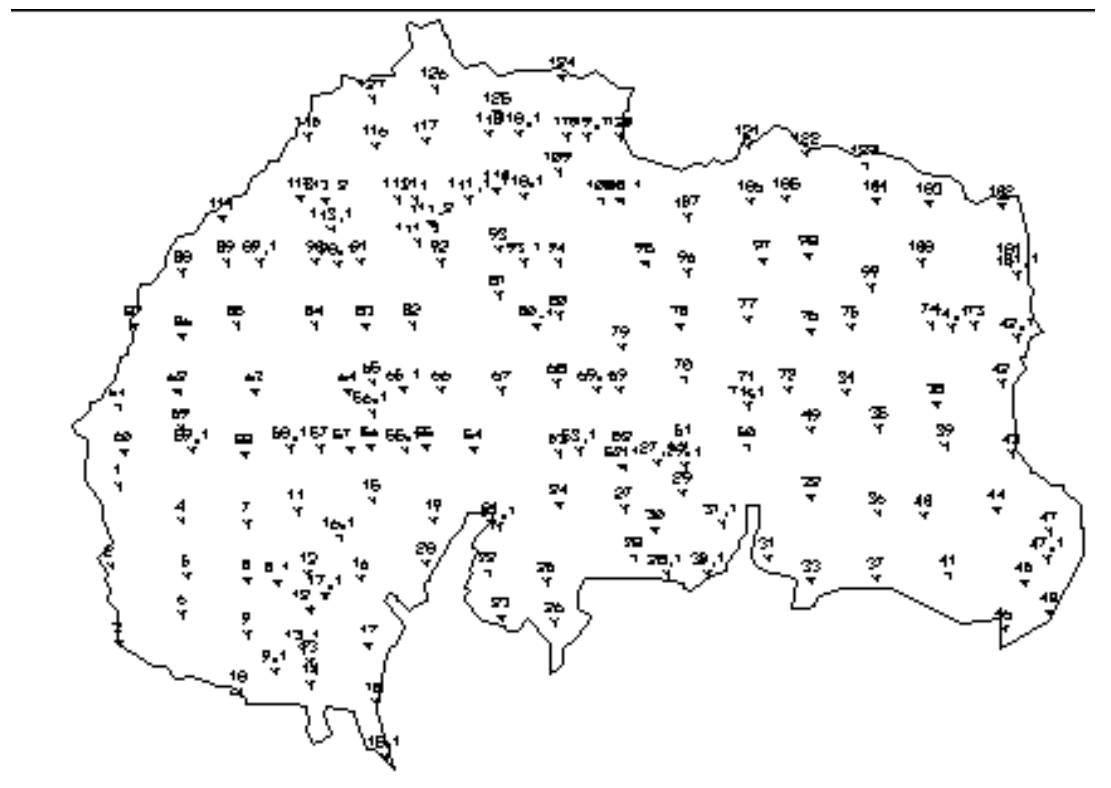

Figura 1: Distribución de los pozos de observación freatimétrica.

Para estimar los semivariogramas se hace uso de la ecuación (27) con $\xi=h$. La figura (2) muestra los 60 semivariogramas experimentales. En esta figura se observa que los puntos de los semivariogramas correspondientes a cada mes, presentan aproximadamente la misma pendiente. En otros términos, puede aceptarse un sólo valor de la potencia $\beta$ de la ecuación (29), sobre el intervalo $0<h<30 \mathrm{~km}$. El número de incógnitas en la ecuación (35) se reduce a $M+1$.

\subsection{Resultados}

Los resultados obtenidos son: i) $\beta \simeq 1,5$ (ecuación 37 ); ii) $D \cong 2,25$ (ecuación 31); y iii) los 60 valores de $c$ (ecuación 38) están en el intervalo: $1,2<c_{j}<1,4$.

La figura (3) presenta la variación de los coeficientes $c_{j}$ para los 60 meses.

La introducción de las variancias de la posición de la superficie del manto $\sigma_{j}^{2}$, permite definir una escala mensual $\left(\lambda_{j}\right)$ para las interdistancias, de tal forma que: $\sigma_{j}^{2}=c_{j} \lambda_{j}^{\beta}$. La 


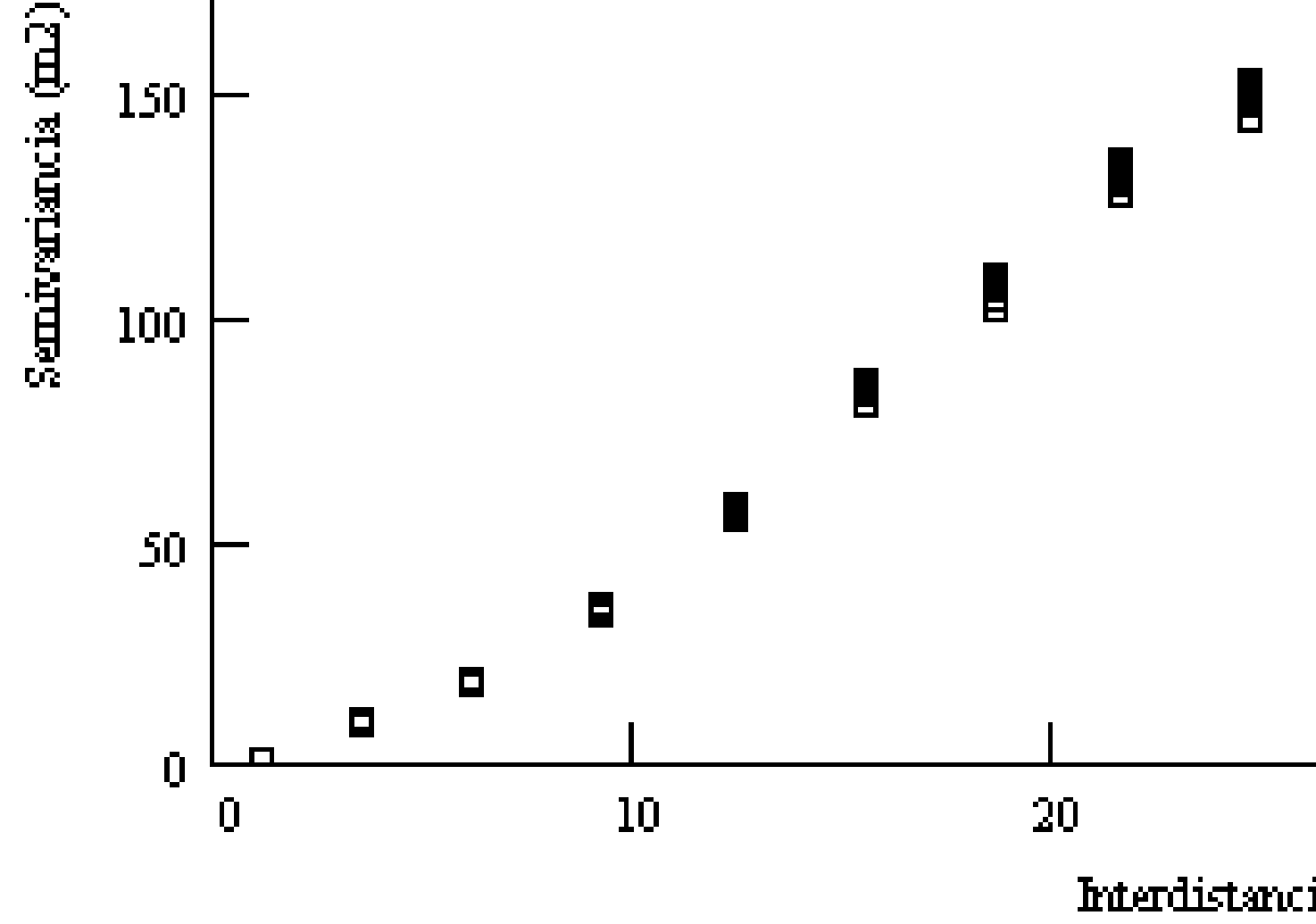

Figura 2: Semivariogramas experimentales para los 60 meses.

ecuación (29) correspondiente a cada mes toma la forma:

$$
\frac{\gamma_{j}(h)}{\sigma_{j}^{2}}=\left(\frac{h}{\lambda_{j}}\right)^{\beta}, j=1,2, \ldots, M .
$$

En escritura adimensional la ecuación (39) se presenta como:

$$
\gamma_{*}=h_{*}^{\beta},
$$

donde $\gamma_{*}=\lambda_{j} / \sigma_{j}^{2}$ y $h_{*}=h / \lambda_{j}$. Los semivariogramas experimentales adimensionales y el semivariograma teórico adimensional se presenta en la figura (4). La función que representa el semivariograma teórico adimensional, con $h_{* c r}=1,29$ y $\gamma_{* c r}=1,46$, es:

$$
\gamma_{*}\left(h_{*}\right)= \begin{cases}h_{*}^{\beta} & 0 \leq h_{*}<h_{* c r} \\ \gamma_{* c r} & h_{*} \geq h_{* c r}\end{cases}
$$




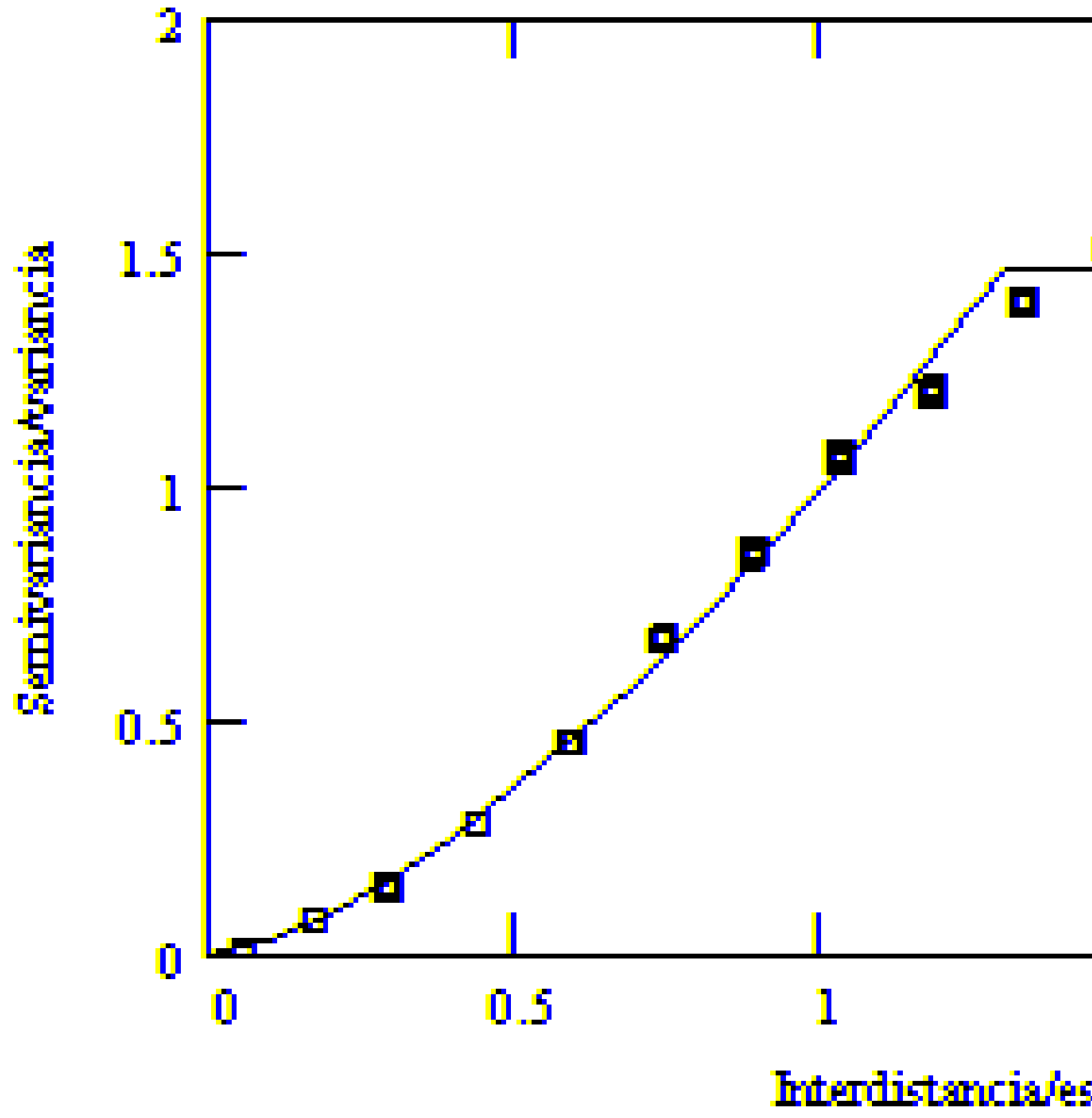

Figura 4: Semivariogramas exp. adimensionales y la curva de ajuste. 


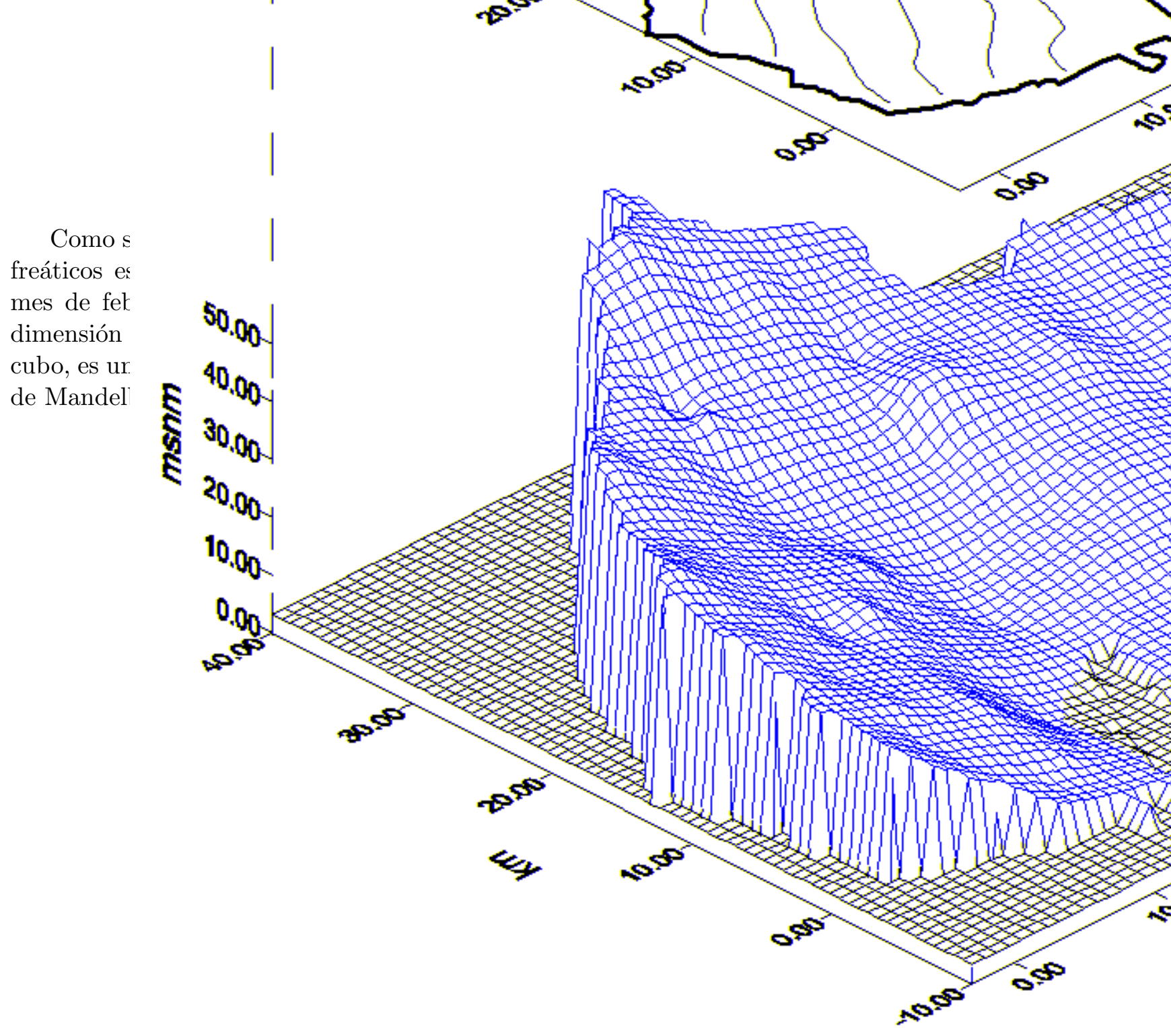

Figura 5: Elevación del nivel freático en febrero de 1995.

\section{Resultados y conclusiones}

Los resultados obtenidos son:

- Se demuestra que la codimensión $D_{T}-D$ del grafo fractal es el exponente de Hölder, (17).

- Para el semivariograma de ajuste se escoge el modelo pontenciado, con el propósito de garantizar la invarianza de escala. La potencia del modelo teórico resulta ser: $\beta \simeq 1,5$ (ecuación 37).

- La dimensión fractal de los mantos freáticos es: ii) $D \cong 2,25$ (ecuación 31).

- La variación de los valores del parámetro $c$, para los 60 meses observados, se ubica en el intervalo: $1,2<c_{j}<1,4$, (ecuación 38). 


\section{Referencias}

[1] Barnsley, M.F. (1993) Fractals Everywhere. Academic Press Professional, Boston.

[2] Colton, D.; Kress, R. (1983) Integrals Equation Methods in Scattering Theory. John Wiley \& Sons, New York.

[3] Daniell, P. J. (1917-1918) "A general form of integral", Ann. Math. 2(19): 279-294.

[4] Falconer, K. (1990) Fractal Geometry. John Wiley \& Sons, New York.

[5] Journel, A.G.; Huijbregts, Ch.J. (1978) Mining Geostatistics, Academic Press, New York.

[6] Kyung, Ch.; Kang, S. (1997) "A practical estimation method for groundwater level elevations", J. Korean Math. Soc. 34(4): 927-947.

[7] Lasota, A.; Myjak, J. (1998) "Semifractals on Polish spaces", Bull. of the Polish Acad. of Scie. Math. 46(2): 179-196.

[8] Luenberger, D.G. (1969) Optimization by Vector Space Methods J. Wiley \& Sons, New York.

[9] Mandelbrot, B. (1983) The Fractal Geometry of Nature. W.H. Freeman and Company, New York.

[10] Mercado, J. R. (1998) El Principio de Causalidad Eficiente en el Análisis Funcional, en preparación.

[11] Oliver, M.A.; Webster, R. (1991) "How geostatiscs can help you", Soil Use and Management $\mathbf{7}(4)$.

[12] Queneau, R. (1990) El lugar de las matemáticas en la clasificación de las ciencias. Introduccción y Selección de Miguel Lara Aparicio, Lecturas Universitarias 7, Antología de Matemáticas, UNAM,.

[13] Rudin, W. (1979) Análisis Real y Complejo. Alhambra, Madrid.

[14] Shilov, G. E.; Gurevich, B. L. (1977) Integral, Measure \& Derivative: A Unified Approach. Dover, Londres. 